\title{
3D Analysis of Porosity in a Ceramic Coating Using X-ray Microscopy
}

\author{
Uta Klement $^{1} \cdot$ Johanna Ekberg $^{1} \cdot$ Stephen T. Kelly $^{2}$
}

Submitted: 30 September 2016/in revised form: 16 January 2017/Published online: 31 January 2017

(c) The Author(s) 2017. This article is published with open access at Springerlink.com

\begin{abstract}
Suspension plasma spraying (SPS) is a new, innovative plasma spray technique using a feedstock consisting of fine powder particles suspended in a liquid. Using SPS, ceramic coatings with columnar microstructures have been produced which are used as topcoats in thermal barrier coatings. The microstructure contains a wide pore size range consisting of inter-columnar spacings, micro-pores and nano-pores. Hence, determination of total porosity and pore size distribution is a challenge. Here, x-ray microscopy (XRM) has been applied for describing the complex pore space of the coatings because of its capability to image the (local) porosity within the coating in $3 \mathrm{D}$ at a resolution down to $50 \mathrm{~nm}$. The possibility to quantitatively segment the analyzed volume allows analysis of both open and closed porosity. For an yttria-stabilized zirconia coating with feathery microstructure, both open and closed porosity were determined and it could be revealed that $11 \%$ of the pore volumes (1.4\% of the total volume) are closed pores. The analyzed volume was reconstructed to illustrate the distribution of open and closed pores in 3D. Moreover, pore widths and pore volumes were determined. The results on the complex pore space obtained by XRM are discussed in connection with other porosimetry techniques.
\end{abstract}

Uta Klement

uta.klement@chalmers.se

1 Department of Materials and Manufacturing Technology, Chalmers University of Technology, 41296 Gothenburg, Sweden

2 Carl Zeiss X-ray Microscopy Inc., 4385 Hopyard Rd, Pleasanton, CA 94588, USA
Keywords porosity $\cdot$ pore size distribution - suspension plasma spray $\cdot$ TBC $\cdot$ topcoat $\cdot$ x-ray microscopy $\cdot$ yttriastabilized zirconia

\section{Introduction}

Generally, porosity degrades the mechanical properties of materials, and in ceramics, the strength is determined by the largest crack-like defect present. Also thermal shock resistance and the thermal insulation capabilities are influenced by the presence of pores and cracks. However, in addition to the amount of porosity, the achievable properties are also influenced by the nature of the individual pores, i.e., whether the porosity consists of fine or coarse pores, their morphologies as well as their relative proportions.

Suspension plasma spraying (SPS) is a recent development in plasma spray technology which has emerged as a means for depositing smaller particles. The process is similar to the atmospheric plasma spraying (APS) process, except that a liquid carrier is used when injecting the powder into the plasma jet. The particles of the liquid suspension used in SPS can be of submicron size, i.e., $<1 \mu \mathrm{m}$ (Ref 1), whereas 10-100 times larger particle are used in APS (Ref 2). The smaller particles are more influenced by the plasma jet flow as it interacts with the substrate (Ref 3) and the difference in droplet flight path leads to formation of a columnar structure (Ref 4, 5). Hence, the coating build-up occurs in a different manner as compared to conventional APS spraying. Yet, thermal barrier coatings (TBCs) produced by SPS have already shown superior properties in terms of thermal properties and thermal shock resistance as compared to their APS counterparts (Ref 6,7). This is mainly due to the inter- 
columnar spacings which improve the strain tolerance of the coating and the presence of pores and micro-cracks which reduce the thermal conductivity (Ref 8 ). But pore size distribution and pore geometry are not only affecting the thermal properties. They also determine to what extent sintering occurs when the TBC is exposed to extreme temperatures $\left(\geq 1200{ }^{\circ} \mathrm{C}\right.$ ) for extended time during operation of the engine ( $\operatorname{Ref} 8,9)$. Again, pore size and shape play an important role as smaller and/or non-sphericalshaped pores have shorter diffusion paths and may therefore close easier when exposed to high temperatures. To optimize the thermal properties and to maintain them during service of the components, it is important to have TBCs with an optimized porosity. In fact, further performance improvements in both lifetime and thermal properties can be envisioned when tailoring the microstructure of the TBC system. Therefore, determination of pore size, pore size distribution, and pore geometry is an essential part of TBC design.

Different techniques commonly used to characterize APS coatings have been evaluated for their ability to determine porosity, i.e., shape and size distribution of pores in SPS coatings (Ref 2). For example, scanning electron microscopy (SEM) can be used to characterize the coating microstructure in cross section. Porosity and crack distribution can be assessed with the help of image analysis from typical images taken at sufficiently high magnification. But images at very high magnifications lead to a loss in global coating information, while too low magnifications are not able to capture the small-scale features like nanometersized pores. Hence, in order to be able to analyze a representative elementary volume of the structure, the resolution is in principle limited to features larger than $\sim 100 \mathrm{~nm}$, which is by far not sufficient to characterize SPS coatings (Ref 2). To improve the results obtained by SEM, 3D imaging by use of focused ion beam (FIB) microscopy and image analysis can be applied to reconstruct pores and cracks including their 3D network. Also nuclear magnetic resonance (NMR) cryoporometry was recently applied to characterize SPS coatings (Ref 10) as the method allows determining porosity and pore size distribution $(5-500 \mathrm{~nm})$ and is also capable of providing information about the pore geometry.

Mercury intrusion porosimetry (MIP) is one of the techniques capable of measuring a large pore size range, theoretically from about $100 \mu \mathrm{m}$ down to a few nanometers, and has been used to quantify pore size distribution in SPS coatings (Ref 11, 12). Also ultra-small angle x-ray scattering (USAXS) can with very high resolution provide information on a wide range of pore sizes for materials with open and closed porosity (Ref 13-15). However, when Marthe et al. (Ref 12) compared porosity measurements obtained by MIP and USAXS, substantial differences in pore size distribution were found. As stated by the authors, the differences are due to the fact that the USAXS technique is suitable to measure nano-pores and does not detect the presence of large heterogeneities, i.e., pores over a few micrometer in size (in fact, it measures pores $<2 \mu \mathrm{m}$ ) while for MIP it is the other way around. This means USAXS is providing deviating results when large pores are numerous and in this case results from MIP are probably more reliable.

Hence, there is growing interest in using other advanced characterization techniques to describe the complex pore space of the coatings at resolutions that allow for quantitative assessment of porosity over a wider pore size range. Here, we focus on investigating a suspension plasmasprayed yttria-stabilized zirconia (YSZ) topcoat by use of $\mathrm{X}$-ray microscopy to assess the fraction and size distribution of open and closed pores in the denser part of the sample within the columnar structure. The analyzed 3D pore space will later-on be compared with the respective sintered material to evaluate which pores are expected to grow/shrink at the intended operating temperature of the engine.

\section{Experimental}

\section{Material}

The TBC system consisted of an YSZ topcoat, a CoNiCrAlY bondcoat and a Hastelloy $\mathrm{X}$ substrate. While the bondcoat was created by high-velocity air-fuel thermal spray process (Uniquecoat, Richmond, USA), the YSZ topcoat was produced by axial SPS using an Axial III highpower plasma torch (Northwest Mettech Corp., Vancouver, Canada). The suspension used as feedstock consisted of 8 wt.\% YSZ dispersed in ethanol (25 wt.\% solid load), and the YSZ powder particles had a median particle size of $500 \mathrm{~nm}$, as stated by the supplier (INNOVNANO, Coimbra, Portugal). The spraying parameters are given in Table 1, and further details about the spray process can be found elsewhere (Ref 16).

\section{Instruments}

The topcoat of the TBC system was analyzed using a Leo 1550 Gemini scanning electron microscope (SEM) equipped with a field emission gun. Prior to the SEM investigations, the specimen was cross-sectioned, polished and gold coated.

Nanoscale 3D x-ray microscopy (XRM) was performed using a ZEISS Xradia 810 Ultra X-ray microscope. Details about the theory and system configuration are provided by Tkachuk et al. (Ref 17) while the use is described by Carl 
Table 1 Spray parameters of topcoat (Ref 16)

\begin{tabular}{lcccc}
\hline Spray distance, $\mathrm{mm}$ & Surface speed, $\mathrm{cm} / \mathrm{s}$ & Power, $\mathrm{kW}$ & Total gas flow, $\mathrm{L} / \mathrm{min}$ & Suspension feed rate, $\mathrm{mL} / \mathrm{min}$ \\
\hline 100 & 216 & 116 & 200 & 45 \\
\hline
\end{tabular}

Zeiss x-ray Microscopy Inc. (Ref 18) and by Merkle and Gelb (Ref 19). Briefly, the Zeiss Xradia Ultra uses an architecture which is conceptually equivalent to that of an optical or transmission electron microscope. The XRM images the specimen at high magnification using $\mathrm{X}$-ray optics, while rotating the specimen in the x-ray beam. The characteristic $\mathrm{K} \alpha \mathrm{x}$-rays $(5.4 \mathrm{keV})$ from a $\mathrm{Cr}$ rotating anode source are focused onto the specimen using a reflective capillary condenser optic. X-rays transmitted by the specimen are imaged using a Fresnel zone plate objective lens and a high-efficiency x-ray detector. An additional phase ring can be placed into the beam path to achieve Zernike phase contrast (Ref 18). The enhanced contrast at edges and boundaries provided by Zernike phase contrast facilitates imaging of internal pores and cracks contained within the specimen.

\section{Data Collection}

\section{Sample Preparation}

Specimens were prepared for nanoscale $\mathrm{x}$-ray imaging by isolating the central portion of a smaller piece of the YSZ topcoat using a ZEISS Auriga focused ion beam (FIB) instrument. The small piece of the topcoat was epoxied onto the end of a steel dowel, and the mounted specimen was loaded into the FIB instrument. By removing the surrounding material using FIB, a pillar of $\sim 80 \mu \mathrm{m}$ in height and $\sim 18 \mu \mathrm{m}$ in diameter was prepared (Fig. 1a). High-resolution (HRES) imaging and large field of view (LFOV) $x$-ray imaging were performed on the same specimen, without further sample trimming or manipulation. In the $2 \mathrm{D} x$-ray projection image in Fig. 1(b), the subvolumes for the LFOV and HRES scans are illustrated with dashed black and dashed white lines, respectively. Moreover, gold spheres of 1.5-3 $\mu \mathrm{m}$ in diameter (Alfa Aesar, USA) were placed on the specimen surface prior to highresolution imaging to serve as fiducial markers during tomographic imaging and aid in image alignment.

\section{Scans and Acquisition Parameters}

Tomographic imaging of each specimen was carried out in both the LFOV and HRES magnification levels. Data were acquired in both absorption contrast and Zernike phase contrast mode for each magnification level. Scan times ranged from 5 to $24 \mathrm{~h}$, depending on the magnification and imaging mode used. An overview of the scan and acquisition parameters is provided in Table 2.

\section{Reconstruction}

Following acquisition of the tomographic data, the 2D radiographs were aligned and reconstructed into $3 \mathrm{D}$ data sets. The resulting 3D data contained voxel sizes of $64 \mathrm{~nm}$ in the LFOV mode and $32 \mathrm{~nm}$ in HRES mode (Table 2).

\section{Interpretation}

In the Zernike phase contrast images, dark to bright intensity fringes highlight the edges and boundaries of the YSZ microstructure. Because of the different refractive indexes between the YSZ and the pore space, the interior of the pores becomes highlighted with a darker outline. On small pores, this creates an effect of darkening the overall intensity of the pore. All renderings and measurements of the $3 \mathrm{D}$ nanoscale $\mathrm{x}$-ray microscopy data were done with the Visual SI Advanced software package (Object Research Systems, Canada).

\section{Results}

\section{Imaging}

The SEM micrographs in Fig. 2 show the investigated YSZ topcoat in cross section. As can be seen, the coating has a columnar, feathery structure. The pores contained in the microstructure are in a wide size range, and inter-columnar spacings/pores are up to several micrometers in diameter (Fig. 2a). At higher magnification, round YSZ particles of various sizes (300 $\mathrm{nm}$ up to $1.5 \mu \mathrm{m}$ ) can be observed in the microstructure, especially in the vicinity of larger pores in the inter-columnar regions (Fig. 2b).

$\mathrm{XRM}$ measurements were performed on the denser part of the YSZ topcoat sample close to the top of the columnar structure. Figure 3 shows 2D slices obtained from the LFOV Zernike phase contrast scan of the YSZ pillar sample. In Fig. 3(a), a virtual slice is taken perpendicular to the long-axis of the pillar, while Fig. 3(b) and (c) shows 


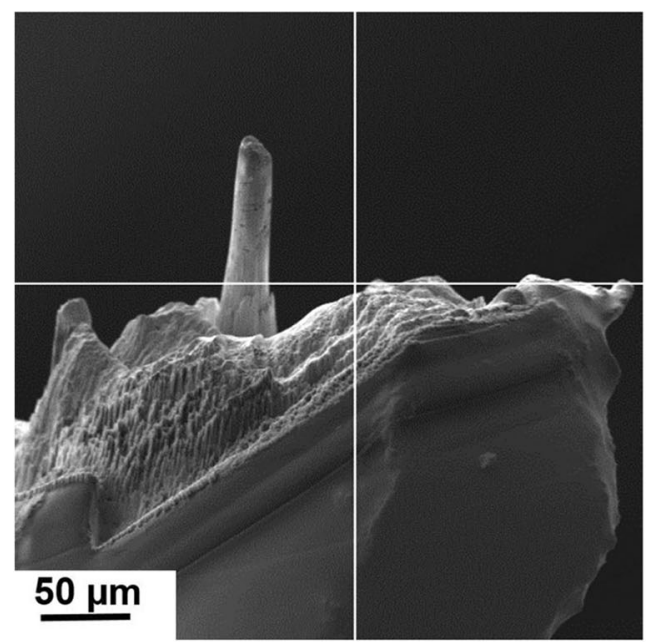

(a)

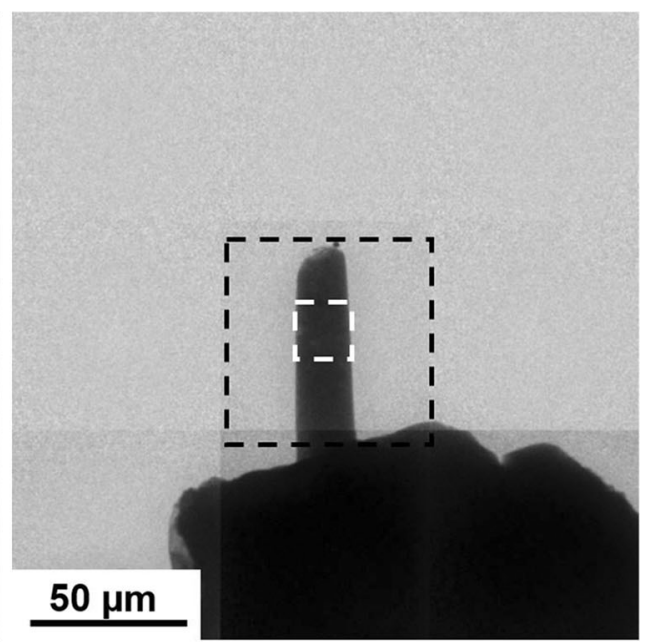

(b)

Fig. 1 (a) SEM micrograph of the pillar sample as prepared by FIB; (b) 2D x-ray projection image of the YSZ sample with the sub-volumes illustrated for LFOV (dashed black line) and HRES (dashed white line) scans

Table 2 Overview of scan and acquisition parameters

\begin{tabular}{lcccc}
\hline Scan & Voxel size, $\mathrm{nm}$ & Field of view (FOV), $\mu \mathrm{m}$ & Imaging mode & Total scan time, $\mathrm{h}$ \\
\hline Large field of view (LFOV) & 64 & 65 & Absorption contrast & 5 \\
Large field of view (LFOV) & 64 & 65 & Zernike phase contrast & 5 \\
High resolution (HRES) & 32 & 16 & Absorption contrast & 16 \\
High resolution (HRES) & 32 & 16 & Zernike phase contrast & 24 \\
\hline
\end{tabular}

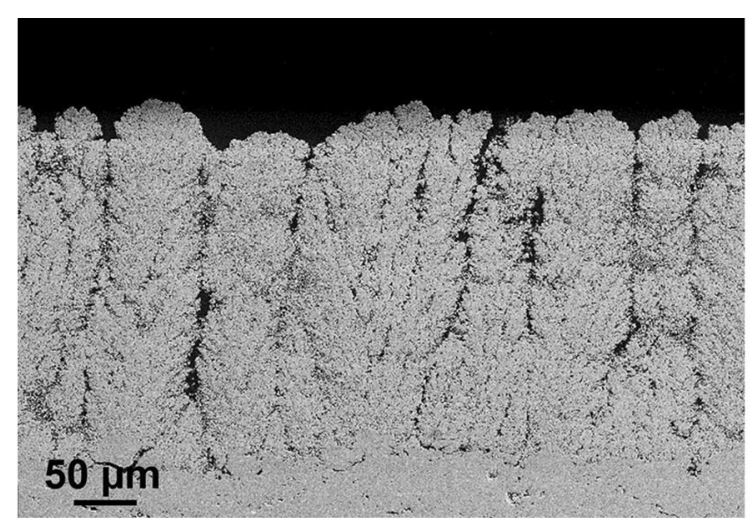

(a)

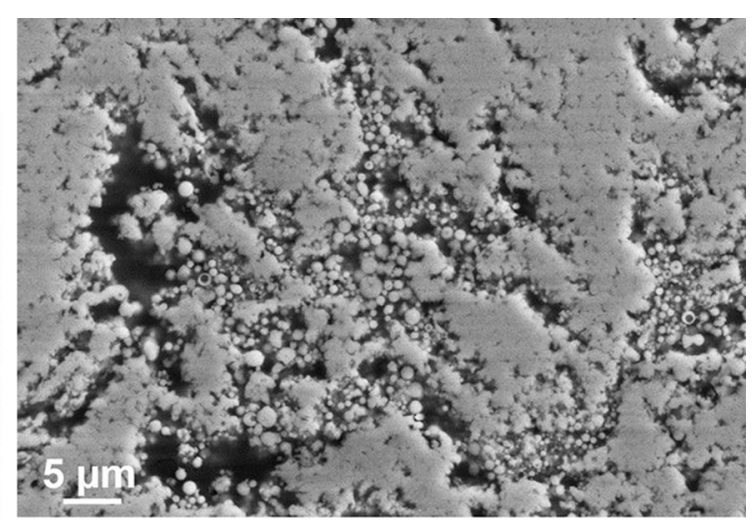

(b)

Fig. 2 SEM micrographs of the YSZ topcoat microstructure: (a) overview showing the columnar, feathery microstructure; (b) image at higher magnification showing round YSZ particles in the vicinity of large pores

virtual slices taken orthogonal to the one shown in Fig. 3(a) (horizontal and vertical cuts as illustrated by the dashed lines and indicated with help of markers, i.e., dots, squares and triangles). Figure 3(d) shows the reconstruction with help of the virtual slices. The markers are included for easier recognition of the virtual slices given in Fig. 3(a), (b), and (c). As can be seen, the pore distribution in the analyzed volume is clearly visible and when using LFOV scan mode and Zernike phase contrast, features/pores with a resolution of down to $150 \mathrm{~nm}$ can be measured. In Fig. 4, the 3D renderings of the HRES and LFOV absorption contrast data sets are given overlaid onto each other. The (faint) boxes show the extent of the two data sets, with the LFOV data clipped away to reveal the HRES data. Figure 4(a) shows the full 


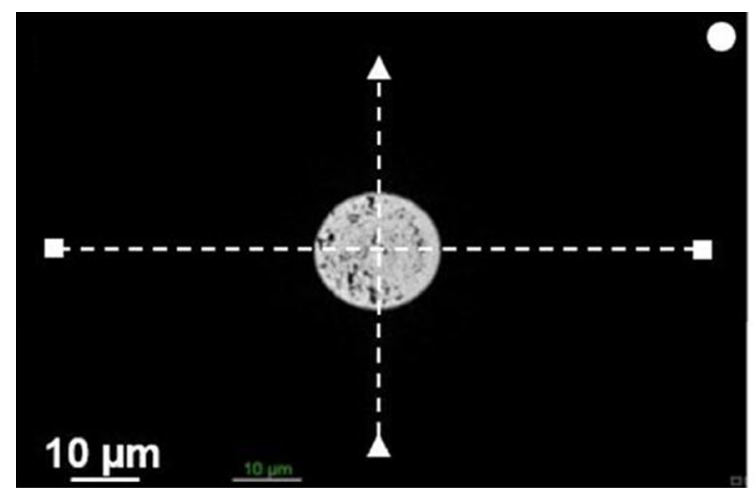

(a)

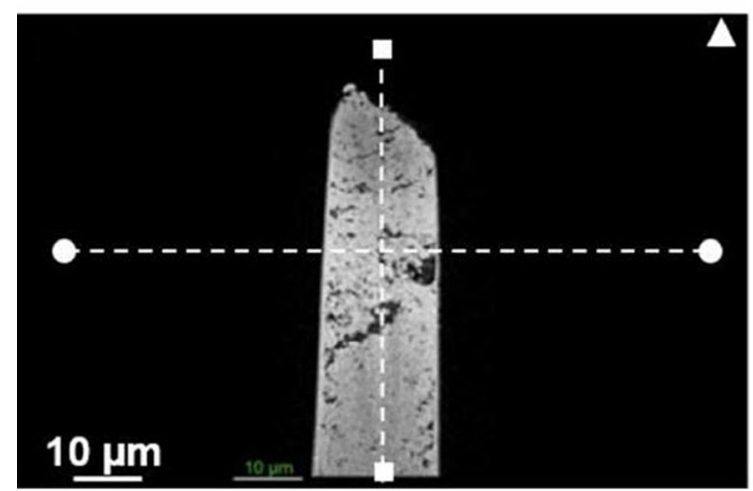

(c)

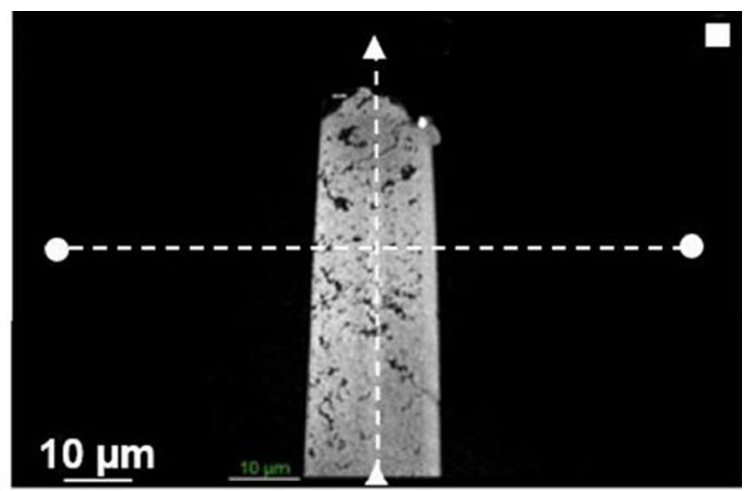

(b)

\section{0}

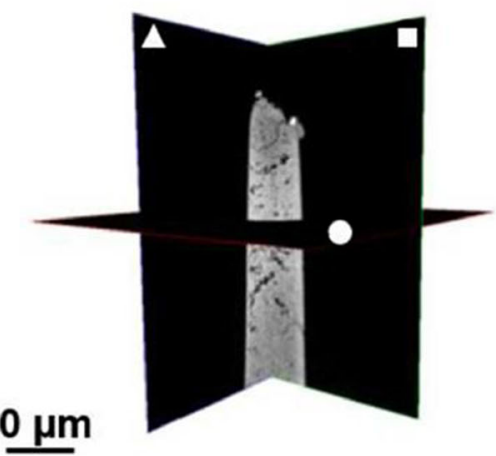

(d)

Fig. 3 (a)-(c) 2D virtual slices obtained from the LFOV Zernike phase contrast scan of the YSZ pillar sample; (d) reconstruction with help of the $2 \mathrm{D}$ virtual slices in (a)-(c)

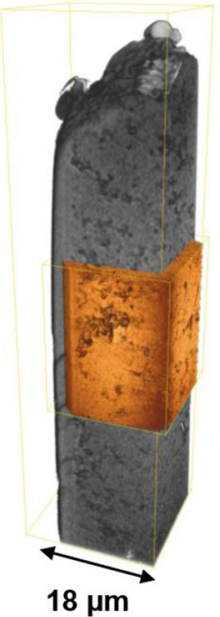

(a)

(b)

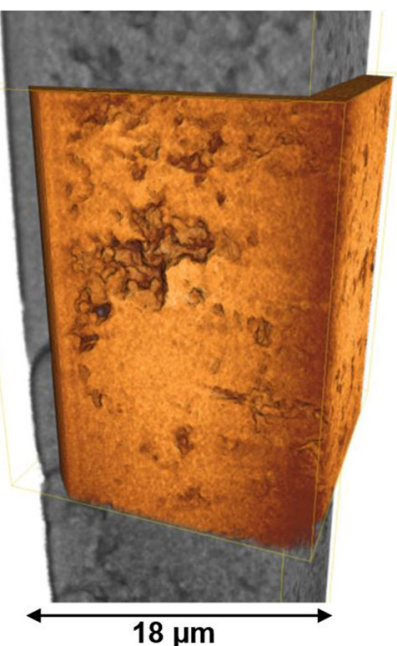

Fig. 4 3D rendering of LFOV and HRES absorption data sets overlaid onto each other (Gold spheres at the top are used as marker): (a) overview and (b) zoomed-in of the HRES data set

volume analyzed (notice the gold spheres at the top of the LFOV data set), whereas Fig. 4(b) provides a zoomed-in view of the HRES data set.
In Fig. 5(a), the reconstructed sample volume imaged in HRES Zernike phase contrast is provided. The 3D subvolume in ultra-HRES Zernike phase contrast shown in Fig. 5(b) was used for detailed analysis, i.e., determination of pore volume and pore widths in the YSZ coating. In the 2D HRES absorption contrast image in Fig. 6, the sizes of different pores and internal features are measured. The smallest pore diameter measured in this case is $60 \mathrm{~nm}$. However, features and pore widths of down to $50 \mathrm{~nm}$ can be resolved in the HRES scan mode.

\section{Fraction of Open and Closed Porosity}

With help of the Visual SI Advanced software by Object Research Systems, Canada, the fraction of open and closed porosity has been determined. Figure 7 shows illustrations of the porosity present in the analyzed volume given in Fig. 5(b). In Fig. 7(a), the 3D ultra-HRES Zernike phase contrast image is partly clipped away to reveal the overlay of the open and closed porosity data (connected open porosity is given in blue and the closed porosity is visualized in red). While Fig. 7(b) presents an overlay of both closed and connected porosity in the analyzed volume, 
Fig. 5 (a) 3D reconstruction of the sample volume imaged in HRES Zernike phase contrast. (Notice the gold sphere at the top used as marker.) (b) 3D subvolume in ultra-HRES Zernike phase contrast which has been used for detailed analysis

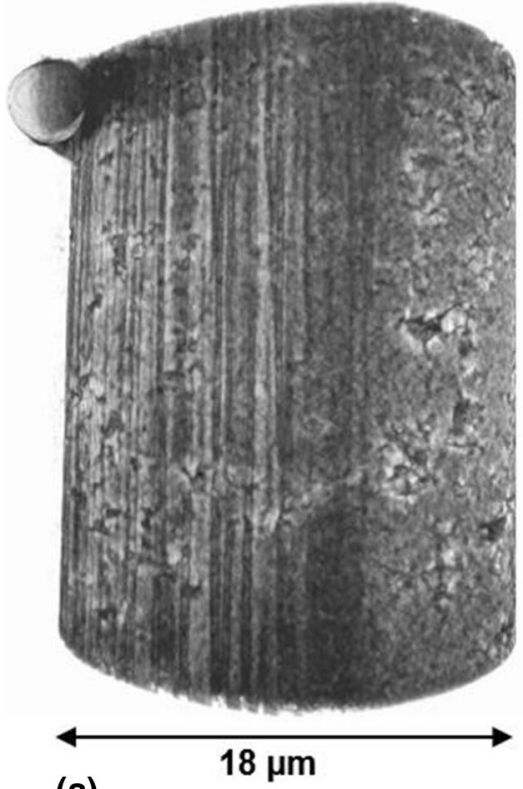

(a)

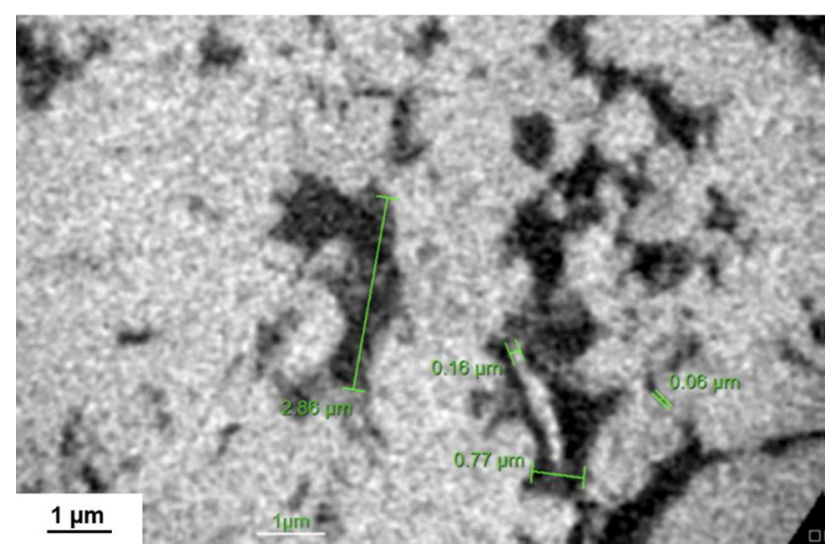

Fig. 6 Ultra-HRES absorption contrast virtual slice image showing measurements on some pores and features

Fig. 7(c) shows only the fraction of closed porosity. Analysis reveals that the analyzed volume contains $99.0 \mu^{3}$ open porosity, which is $89 \%$ of the measured pore volume and $11.6 \%$ of the total volume. In comparison, the closed pores have a volume of $12.1 \mu \mathrm{m}^{3}$. This amounts to $11 \%$ of the pore volume and $1.4 \%$ of the total volume. Hence, the analyzed volume has a total porosity of $13 \%$.

\section{Pore Volume}

Closed pores larger than $3 \times 3 \times 3$ voxels (Fig. 7c) were analyzed for volume with help of the Visual SI Advanced software by Object Research Systems, Canada. Figure 8 displays the distribution of these pores, with volumes corresponding to the color legend below (between 0 and

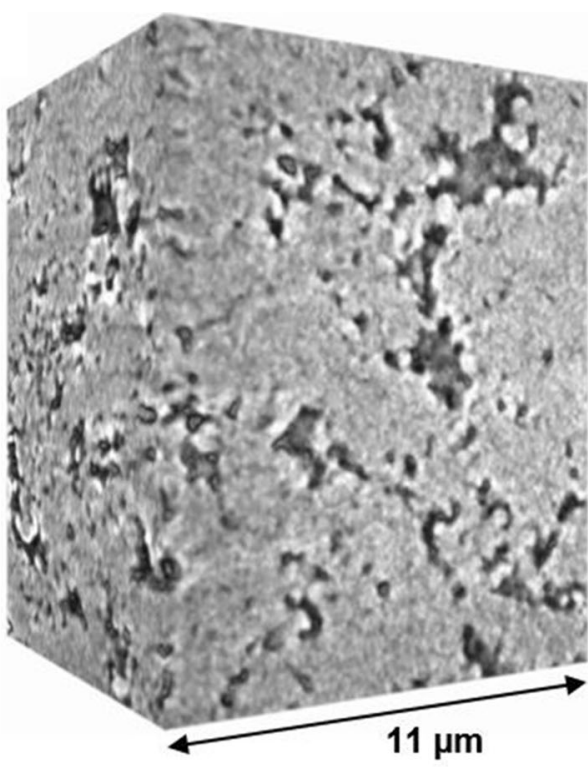

(b)

$0.7607 \mu \mathrm{m}^{3}$ with a step size of $0.0951 \mu \mathrm{m}^{3}$ ). As can be seen, the analyzed volume contains a large amount of small closed pores $\left(<0.09 \mu \mathrm{m}^{3}\right.$ given in blue in Fig. 8). This is also visible in the pore volume histogram of the closed pores (Fig. 9) which has an average pore volume of $0.012 \mu \mathrm{m}^{3}$.

\section{Discussion}

XRM allows nondestructive analysis of samples, and with help of common reconstruction software, it is possible to obtain a three-dimensional view of the analyzed sample area and perform virtual cuts through the analyzed volume at desired locations. Using the ZEISS Xradia 810 Ultra, the pore space of a columnar feathery YSZ topcoat was investigated and open and closed pore sets were isolated and separately analyzed. This analysis revealed a total porosity of $13 \%$ of which $89 \%$ were open connected pores and $11 \%$ closed pores. Using virtual slices of the analyzed volume, the width of pores was measured down to a resolution of $50 \mathrm{~nm}$. The volume of the closed pores was also analyzed, and an average pore volume of $0.012 \mu \mathrm{m}^{3}$ was obtained. Hence, XRM has shown to be a suitable technique for determining porosity in coatings produced by SPS.

In comparison with other porosimetry techniques like MIP which is suitable for measuring porosity over a wide pore size range down to nanometers, XRM has the advantage of being an imaging technique which allows to reconstruct the analyzed volume. Hence, a 3D reconstruction of the pore space is achievable which, in addition to 


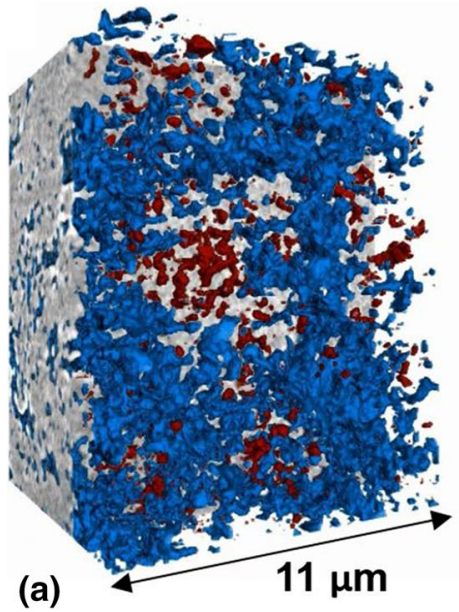

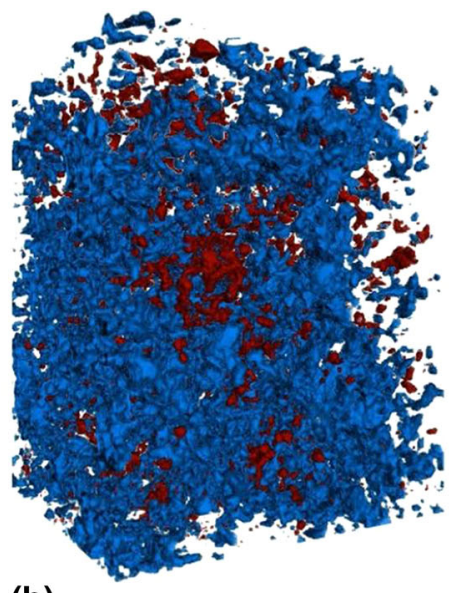

(b)

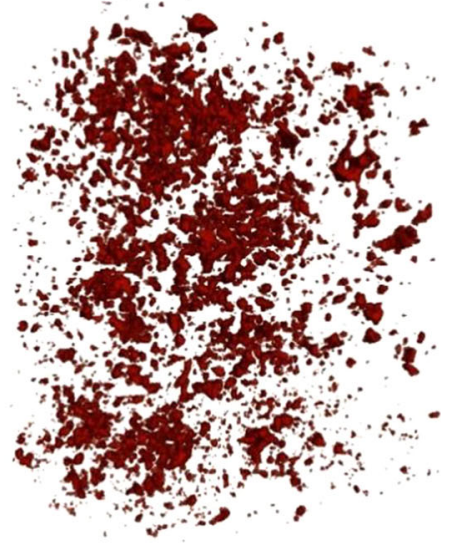

(c)

Fig. 7 Illustrations of the porosity present in the analyzed volume: (a) hybrid of analyzed volume and closed and connected porosity; (b) overlay of closed and connected porosity; (c) fraction of closed porosity present in the analyzed volume

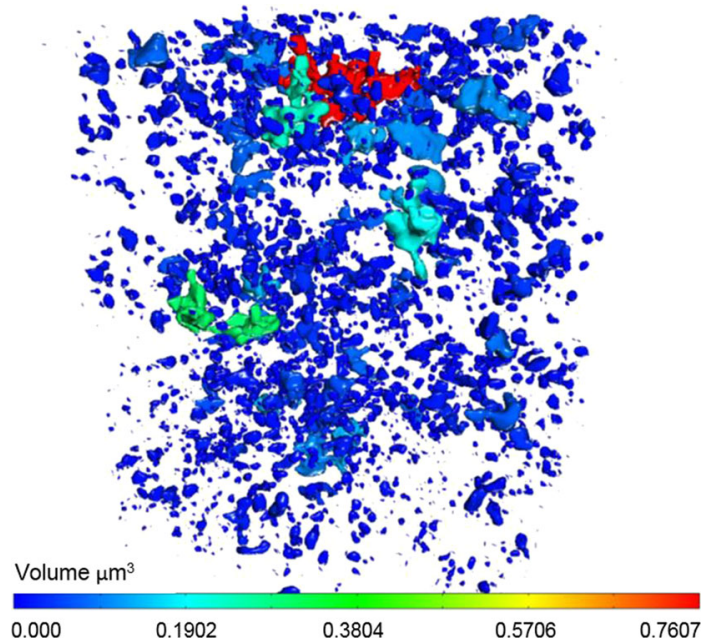

Fig. 8 Distribution of the pore volumes of the closed pores larger than $3 \times 3 \times 3$ voxels in the 3D sub-volume in HRES Zernike phase contrast (the volumes correspond to the color legend which ranges between 0 and $0.7607 \mu \mathrm{m}^{3}$ with a step size of $0.0951 \mu \mathrm{m}^{3}$ )

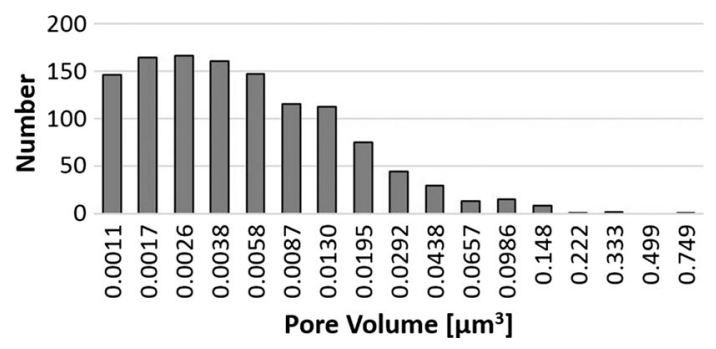

Fig. 9 Pore volume histogram of the closed pores larger than $3 \times 3 \times 3$ voxels in the $3 \mathrm{D}$ sub-volume in HRES Zernike phase contrast (Fig. 8)

dedicated analysis, enables a visual impression of the complex pore shapes. Furthermore, the XRM technique is nondestructive (the sample remains intact following the measurement), allowing further analysis after subjecting the sample to processing steps such as thermal cycling and opening the door for evolutionary studies of sample microstructure. FIB milling in combination with SEM imaging also allows for reconstruction of similar volumes at similar or higher resolution (open and closed pores could be analyzed at submicron to nanometer resolution Ref 20); however, the technique is destructive and problems with charging of the non-conductive sample may occur during the measurements (Ref 21). As for scattering techniques, the capability of USAXS in measuring very small pore sizes is unprecedented, but the technique reveals no information about the spatial distribution of the pore space within the material and the technique is not readily available for routine/in-house measurements. It needs to be mentioned that the volume analyzed by use of XRM is limited and only local information on open and closed pores in form of size and shape is obtained (in 3D and at high resolution). Total porosity of the coating which would include nanometer-sized pores and the large inter-columnar spacings is not accessible in this way. Hence, far from replacing the earlier methods (even though the use of MIP is more and more restricted out of environmental reasons), newer approaches/techniques such as XRM provide complementary data that extend our ability to quantitatively describe the pore space in SPS coatings. To be able to optimize the thermal properties and to maintain them during service of the components, it will be important to make readily use of such advanced characterization techniques in TBC design.

Acknowledgments Stefan Björklund and Ashish Ganvir at University West, Trollhättan, Sweden, are acknowledged for providing the TBC sample. The authors would like to thank Västra Götalandsregionen for funding in connection with the PROSAM project. 
Open Access This article is distributed under the terms of the Creative Commons Attribution 4.0 International License (http://crea tivecommons.org/licenses/by/4.0/), which permits unrestricted use, distribution, and reproduction in any medium, provided you give appropriate credit to the original author(s) and the source, provide a link to the Creative Commons license, and indicate if changes were made.

\section{References}

1. Mettech Northwest Mettech Corp., Axial III Suspension Plasma Spray. http://www.mettech.com/coating-technology/axial-IIIplasma-spray.php, 2016. Accessed 29 June 2016

2. P. Fauchais and A. Vardelle, Solution and Suspension Plasma Spraying of Nanostructure Coatings, Advanced Plasma Spray Applications, H. Jazi, Ed., InTech, Chennai, 2012, p 149-188

3. O. Berghaus, S. Bouaricha, J.-G. Legoux, C. Moreau, and T. Chraska, Suspension Plasma Spraying of Nano-ceramics Using an Axial Injection Torch, Thermal Spray 2005: Thermal Spray Connects: Explore Its Surfacing Potential!, E. Lugscheider, Ed., May 2-4, 2005 (Basel, Switzerland), DVS-German Welding Society, 2005, p 1434-1440

4. K. Van Every, M.J.M. Krane, R.W. Trice, H. Wang, W. Porter, M. Besser, D. Sordelet, J. Ilavsky, and J. Almer, Column Formation in Suspension Plasma-Sprayed Coatings and Resultant Thermal Properties, J. Therm. Spray Technol., 2011, 20(4), p 817-828

5. P. Sokołowski, L. Pawłowski, D. Dietrich, T. Lampke, and D. Jech, Advanced Microscopic Study of Suspension PlasmaSprayed Zirconia Coatings with Different Microstructures, $J$. Therm. Spray Technol., 2016, 25(1-2), p 94-104

6. N. Curry, K. VanEvery, T. Snyder, and N. Markocsan, Thermal Conductivity Analysis and Lifetime Testing of Suspension Plasma-Sprayed Thermal Barrier Coatings, Coatings, 2014, 4(3), p 630-650

7. P. Fauchais, M. Vardelle, A. Vardelle, and S. Goutier, What Do We Know, What are the Current Limitations of Suspension Plasma Spraying?, J. Therm. Spray Technol., 2015, 24(7), p 1120-1129

8. N. Curry, "Design of Thermal Barrier Coatings," Ph.D. thesis, University West, 2014

9. I.O. Golosnoy, A. Cipitria, and T.W. Clyne, Heat Transfer Through Plasma-Sprayed Thermal Barrier Coatings in Gas Turbines: A Review of Recent Work, J. Therm. Spray Technol., 2009, 18(5-6), p 809-821

10. J. Ekberg, L. Nordstierna, and U. Klement, Porosity Investigation of Thermal Barrier Topcoats Using NMR Cryoporometry, to be published
11. A. Portinha, V. Teixeira, J. Carneiro, J. Martins, M.F. Costa, R. Vassen, and D. Stoever, Characterization of Thermal Barrier Coatings with a Gradient in Porosity, Surf. Coat. Technol., 2005, 195(2-3), p 245-251

12. J. Marthe, E. Meillot, G. Jeandel, F. Enguehard, and J. Ilavsky, Enhancement of Scattering and Reflectance Properties of PlasmaSprayed Alumina Coatings by Controlling the Porosity, Surf. Coat, Technol., 2013, 220, p 80-84

13. A. Bacciochini, G. Montavon, J. Ilavsky, A. Denoirjean, and P. Fauchais, Porous Architecture of SPS thick YSZ Coatings Structured at the Nanometer Scale $(\sim 50 \mathrm{~nm})$, J. Therm. Spray Technol., 2010, 19(1-2), p 198-206

14. A. Bacciochini, F. Ben-Ettouil, E. Brousse, J. Ilavsky, G. Montavon, A. Denoirjean, S. Valette, and P. Fauchais, Quantification of Void Networks of As-Sprayed and Annealed Nanostructured Yttria-Stabilized Zirconia (YSZ) Deposits Manufactured by Suspension Plasma Spraying, Surf. Coat. Technol., 2010, 205(3), p 683-689

15. A. Bacciochini, J. Ilavsky, G. Montavon, A. Denoirjean, F. Benettouil, S. Valette, P. Fauchais, and K. Wittmann-teneze, Quantification of Void Network Architectures of Suspension PlasmaSprayed (SPS) Yttria-Stabilized Zirconia (YSZ) Coatings Using Ultra-small-Angle X-ray Scattering (USAXS), Mater. Sci. Eng. $A, 2010, \mathbf{5 2 8}(1), \mathrm{p}$ 91-102

16. A. Ganvir, N. Curry, N. Markocsan, P. Nylén, S. Joshi, M. Vilemova, and Z. Pala, Influence of Microstructure on Thermal Properties of Axial Suspension Plasma-Sprayed YSZ Thermal Barrier Coatings, J. Therm. Spray Technol., 2016, 25(1-2), p 202212

17. A. Tkachuk, F. Duewer, H. Cui, M. Feser, S. Wang, and W. Yun, $\mathrm{X}$-ray Computed Tomography in Zernike Phase Contrast Mode at $8 \mathrm{keV}$ with 50-nm Resolution Using $\mathrm{Cu}$ Rotating Anode X-ray Source, Zeitschrift fur Krist, 2007, 222(11), p 650-655

18. Carl Zeiss X-ray Microscopy, Inc., X-ray Nanotomography in the Laboratory with ZEISS Xradia Ultra 3D X-ray Microscopes, White Paper, http://pages.microscopy.zeiss.com/X-ray-Nanotomographyin-the-Laboratory-with-ZEISS-Xradia-Ultra.html. Accessed 26 June 2016

19. A.P. Merkle and J. Gelb, The Ascent of 3D X-ray Microscopy in the Laboratory, Micros. Today, 2013, 21(02), p 10-15

20. D.R. Cole and M.A. Arthur, Unconventional Hydrocarbon Resources: Prospects and Problems, Elements, 2014, 10(4), p 257-264

21. L.M. Anovitz and D.R. Cole, Characterization and Analysis of Porosity and Pore Structures, Mineral. Geochem., 2015, 80, p 61164 